\title{
Plasma levels of microRNA-21, -126 and $-423-5 p$ alter during clinical improvement and are associated with the prognosis of acute heart failure
}

\author{
STÉFANIE INGRID DOS REIS SCHNEIDER ${ }^{1,5^{*}}$, DAIANE SILVELLO ${ }^{1 *}$, NIDIANE CARLA MARTINELLI ${ }^{1}$, \\ ARTHUR GARBIN $^{1}$, ANDRÉIA BIOLO ${ }^{1-3}$, NADINE CLAUSELL ${ }^{1-3}$, MICHAEL ANDRADES $^{1}$, \\ KÁTIA GONÇALVES DOS SANTOS ${ }^{1,4}$ and LUÍS EDUARDO ROHDE ${ }^{1-3}$ \\ ${ }^{1}$ Cardiovascular Experimental and Molecular Laboratory, Experimental Research Center; \\ ${ }^{2}$ Heart Failure and Cardiac Transplant Unit, Cardiology Division, Hospital de Clínicas de Porto Alegre, \\ Porto Alegre, RS 90035-903; ${ }^{3}$ Department of Internal Medicine, Medical School, \\ Universidade Federal do Rio Grande do Sul, Porto Alegre, RS 90040-341; ${ }^{4}$ Laboratory of \\ Human Molecular Genetics, Universidade Luterana do Brasil, Canoas, RS 92425-900, Brazil
}

Received August 25, 2017; Accepted November 9, 2017

DOI: $10.3892 / \mathrm{mmr} .2018 .8428$

\begin{abstract}
MicroRNAs are associated with myocardial damage and heart failure (HF). The present study investigated whether the plasma levels of microRNA (miR)-21, -126 and $-423-5 p$ alter according to the (de)compensated state of patients with $\mathrm{HF}$ and are associated with all-cause mortality. In 48 patients with HF admitted to the emergency room for an episode of acute decompensation, blood samples were collected to measure miR and B-type natriuretic peptide levels within $24 \mathrm{~h}$ of hospital admission, at the time of hospital discharge, and a number of weeks post-discharge (chronic stable compensated state). Levels of miR-21, miR-126 and miR-423-5p increased between admission and discharge, and
\end{abstract}

Correspondence to: Professor Kátia Gonçalves dos Santos, Laboratory of Human Molecular Genetics, Universidade Luterana do Brasil, Prédio 22, Avenue Farroupilha, 8001, $5^{\circ}$ andar, Canoas, RS 92425-900, Brazil

E-mail:kgsantos2010@gmail.com

Present address: ${ }^{5}$ Centro Universitário Ritter dos Reis - UniRitter, Campus Canoas, Rua Santos Dumont, 888, Canoas, RS 92120-110, Brazil

*Contributed equally

Abbreviations: $\mathrm{HF}$, heart failure; miR, microRNA; BNP, B-type natriuretic peptide; LV, left ventricular; NYHA, New York Heart Association; ADHF, acute decompensated heart failure; LVEF, left ventricular ejection fraction; RT-qPCR, reverse transcription-quantitative polymerase chain reaction; HCPA, Hospital de Clínicas de Porto Alegre

Key words: biomarker, cardiovascular disease, heart failure, miR, prognosis, survival decreased following clinical compensation. During follow-up (up to 48 months), 38 patients (79\%) were rehospitalized at least once and 21 patients (44\%) succumbed. Patients who had increased levels of miR-21 and miR-126 at the time of clinical compensation exhibited better 24-month survival and remained rehospitalization-free for a longer period compared with those with low levels. Additionally, patients whose levels of miR-423-5p increased between admission and clinical compensation experienced fewer hospital readmissions in the 24 months following the time of clinical compensation compared with those who had decreased levels. It was concluded that the plasma levels of miR-21, miR-126 and miR-423-5p altered during clinical improvement and were associated with the prognosis of acute decompensated HF.

\section{Introduction}

MicroRNAs (miRs) are small endogenous non-coding RNA molecules that regulate essential mechanisms for cardiovascular development and function, including cell growth, differentiation, proliferation and apoptosis (1-3). Dysregulated expression of specific microRNAs leads to the reactivation of fetal genes, left ventricular (LV) remodeling and cardiac hypertrophy in the failing heart $(1,4)$. As circulating microRNAs are stable and likely reflect myocardial damage and ventricular remodeling, they may be useful as diagnostic or prognostic biomarkers for cardiovascular disorders, including heart failure (HF) (4-6).

Studies have focused on the potential application of circulating microRNAs in the diagnosis of HF (6). Their prognostic value was evaluated in clinically stable $(7,8)$ and decompensated (7) outpatients with $\mathrm{HF}$, and in patients with HF who improved from New York Heart Association (NYHA) class IV to class III (9). A number of investigators have additionally reported on the association between circulating miR levels and short-term outcomes in patients with acute decompensated HF (ADHF) (10-13). Based on findings 
reported by Qiang et al (8), Fukushima et al (9), Fan et al (14), Goren et al (15) and Tijsen et al (16), and considering that episodes of ADHF are associated with transient increases in the blood levels of markers of cardiac myocyte injury, extracellular matrix turnover, and inflammation (17-19), possibly reflecting an acceleration of pathological myocardial remodeling (20), it was decided to measure the plasma levels of miR-21,-126 and -423-5p in patients with ADHF at the time of hospital admission to determine whether they alter during clinical improvement. The present study additionally investigated whether the levels of these three miRs were associated with all-cause mortality for a longer follow-up period (up to 48 months).

\section{Materials and methods}

Study population. A total of 60 adult patients (age $\geq 18$ years) with ADHF admitted to the emergency room of the Hospital de Clínicas de Porto Alegre (HCPA; Porto Alegre, Brazil) between May 2011 and June 2012 were prospectively enrolled within $24 \mathrm{~h}$ of admission. Inclusion criteria were a previous diagnosis of $\mathrm{HF}$ and impaired LV systolic function with a LV ejection fraction (LVEF) $\leq 45 \%$, defined by transthoracic echocardiography. The diagnosis of ADHF with volume overload was defined clinically by the presence of worsening symptoms of dyspnea, paroxysmal nocturnal dyspnea, and/or orthopnea in conjunction with clinical signs of circulatory congestion (elevated jugular venous pressure, hepatojugular reflux, hepatomegaly and/or peripheral edema). Exclusion criteria were as follows: Pregnancy, dialysis, moderate to severe aortic stenosis, active malignancy, significant renal (creatinine $>265.2 \mu \mathrm{mol} / \mathrm{l}$ ) or hepatic (cirrhosis or active hepatitis) dysfunction, and moderate to severe rheumatic disease. Patients with concomitant acute coronary syndromes within the previous three months or severe hemodynamic instability requiring intravenous vasoactive drugs were excluded. In addition, patients for whom it was not possible to collect at least one additional blood sample after admission $(n=12)$ were excluded.

The present study additionally included 17 healthy controls without LV dysfunction from the Hemotherapy Division of HCPA between February 2013 and February 2015 to constitute a reference group for the determination of the relative expression levels of miRs (mean age, $59 \pm 10$ years; 12 males; 16 Caucasians) (21). The Research Ethics Committee of HCPA approved the research protocol and all subjects provided written informed consent (Institutional Review Board no. 0000921; study nos. 11-0016 and 12-0084).

Data and sample collection. Following enrollment, demographic, clinical history, comorbidity, echocardiographic, electrocardiographic and laboratory data were collected by reviewing the patient records. To measure the plasma levels of B-type natriuretic peptide (BNP) and miRs, blood samples were collected within the first $24 \mathrm{~h}$ of admission at emergency, at the time of hospital discharge, and a number of weeks post-discharge (a mean of $4 \pm 2$ months). The blood sample at the third time point was collected during an outpatient visit as part of routine clinical care from those who had returned to a chronic stable compensated state (clinical compensation). The criteria for chronic stable compensation were a lack of evidence of volume overload by clinical examination, no current need for diuretic adjustment and no hospital admission for ADHF within the previous two months.

Of the 60 patients admitted to emergency service and initially included in the study, 42 had the second sample collected at the time of hospital discharge and 41 had the third sample collected a number of weeks after discharge, in the chronic compensated state. In total, 48 patients had at least two blood samples collected. Among them, 27 had the blood samples collected at the three time points. The reasons for missing patients during the follow-up included logistical problems collecting blood samples at discharge, new episodes of HF decompensation, lack of attendance at regular consultations, and mortality. Therefore, the findings reported in the present study are the result of the data analysis of 48 patients from whom at least two blood samples were taken (Fig. 1).

\section{Blood sample preparation and measurement of BNP and miR} levels. All laboratory analyses were performed by investigators blinded to clinical data. Blood samples were collected in EDTA-containing tubes and plasma was isolated by centrifugation at $453 \mathrm{x}$ g for $15 \mathrm{~min}$ at $4^{\circ} \mathrm{C}$ within $1 \mathrm{~h}$ following collection. Plasma samples were frozen at $-70^{\circ} \mathrm{C}$ until the assays were performed and subjected to one freeze-thaw cycle. Levels of BNP were measured using a commercially available immunoassay kit (Advia Centaur BNP System; Siemens AG, Munich, Bavaria, Germany; cat. no. 02816138).

miRs were isolated from $495 \mu \mathrm{l}$ plasma using the mirVana PARIS kit with enrichment for small RNAs (Ambion; Thermo Fisher Scientific, Inc., Waltham, MA, USA). Following protein denaturation, $50 \mathrm{pM}$ synthetic miR-39 from Caenorhabditis elegans (cel-miR-39; Qiagen, Inc., Valencia, CA, USA) was spiked-in (fixed volume of $5 \mu$ l) to plasma samples to control for technical variations throughout the RNA isolation and quantitative procedures. Total RNA concentration was determined by spectrophotometry (NanoDrop 1000; Thermo Fisher Scientific, Inc., Wilmington, DE, USA).

Plasma levels of miR-21, -126 and $-423-5 p$ were measured by reverse transcription-quantitative polymerase chain reaction (RT-qPCR) on the 7500 Real Time PCR System (Life Technologies; Thermo Fisher Scientific, Inc.). Reverse transcription reactions were performed using a TaqMan ${ }^{\circledR} \mathrm{miR}$ RT kit (Life Technologies; Thermo Fisher Scientific, Inc.). The reaction mixtures for cDNA synthesis were incubated for $30 \mathrm{~min}$ at $16^{\circ} \mathrm{C}, 30 \mathrm{~min}$ at $42^{\circ} \mathrm{C}$, and $5 \mathrm{~min}$ at $85^{\circ} \mathrm{C}$, and then held at $4^{\circ} \mathrm{C}$. The PCR amplification reactions were run in triplicate using pre-designed TaqMan ${ }^{\circledR}$ MicroRNA Assays, containing specific primers and probes, according to the manufacturer's protocol (Life Technologies; Thermo Fisher Scientific, Inc.; cat. no. 4427975, assay ID nos. 000200, 000397, 002228 and 002340 for cel-miR-39, hsa-miR-21, hsa-miR-126 and hsa-miR-423-5p, respectively). The PCR conditions were $95^{\circ} \mathrm{C}$ for $10 \mathrm{~min}$ followed by 40 cycles of amplification $\left(95^{\circ} \mathrm{C}\right.$ for $15 \mathrm{sec}$ and $60^{\circ} \mathrm{C}$ for $60 \mathrm{sec}$ ). Relative expression levels for each microRNA were estimated using the $2^{-\Delta \Delta C q}$ method (22) using cel-miR-39 as the reference gene and healthy controls as the reference group.

Study end points. Primarily, the present study assessed whether the expression of miR-21, miR-126 and miR-423-5p 


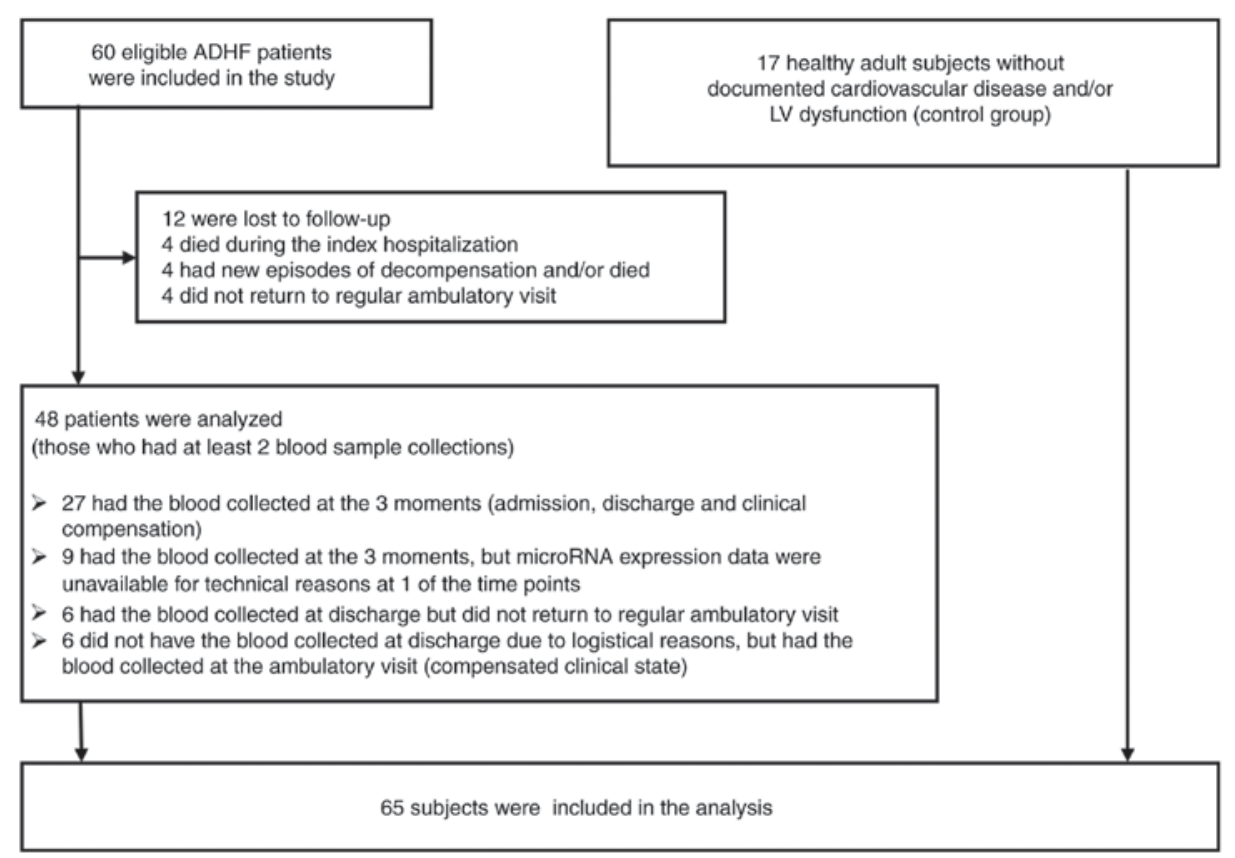

Figure 1. Flowchart of study recruitment illustrating the number of subjects involved at each stage.

differed according to the (de)compensated state of patients with HF by comparing the plasma levels among the three time points. Secondly, the present study evaluated the correlation between plasma levels of BNP and miRs. Thirdly, the association between the selected miRs with hospital readmission and all-cause mortality within 24 and 48 months was evaluated. These outcomes were verified by reviewing the hospital registry or by telephone contact. Survival data were last updated in August 2016.

Statistical analysis. Categorical variables are reported as the absolute numbers and percentages. Continuous variables are expressed as the mean \pm standard deviation, mean \pm standard error of the mean, or as the median (25th and 75 th percentile). Plasma levels of BNP and miRs at admission, at discharge and following clinical compensation were compared by the generalized estimation equation, and P-values were adjusted for pairwise comparisons by Bonferroni correction. To compare the microRNA levels of healthy controls with those estimated for patients with $\mathrm{HF}$ at each time point, the Kruskal-Wallis test was used followed by Dunn's post-hoc test. The Shapiro-Wilk test was used to verify whether continuous variables had a normal distribution. For the comparison of means between two groups and for the correlation analyses, the values of non-normally distributed variables were log-transformed or converted to the square root. Differences between quantitative variables across binary clinical categories were assessed using the Student's t-test or the Mann-Whitney test for the variables that were unable to be normalized (sodium, number of hospital readmissions, and $\Delta-\mathrm{miR}$, corresponding to the alteration in miR levels at clinical compensation compared with admission). Categorical variables were compared between groups of patients with the $\chi^{2}$ test or Fisher's exact test.

Kaplan-Meier survival analysis was performed to evaluate the association between BNP and miRs with all-cause mortality within 24 and 48 months, by grouping the patients in two categories according to whether they had levels above or below the median. Survival curves were constructed considering the period between the date of the first admission and the last registry of follow-up or mortality, and were compared using the log-rank test. Statistical analyses were performed with the use of the SPSS statistical package (version 18.0) for Windows (SPSS, Inc., Chicago, IL, USA) or GraphPad Prism (version 6.01) for Windows (GraphPad Software Inc., La Jolla, CA, USA). Two-tailed $\mathrm{P}<0.05$ was considered to indicate a statistically significant difference.

\section{Results}

Baseline characteristics of study population. The baseline characteristics of the 48 patients with ADHF included in the present study are presented in Table I. The majority of patients were middle-aged Caucasian males with severe LV dysfunction and non-ischemic etiology (hypertensive, alcoholic, valvular or myocarditis). Upon admission for ADHF, all patients were diagnosed as NYHA class III or IV. The primary causes of the acute decompensation episodes were infection (50\%) and poor adherence to treatment $(35 \%)$. The presence of comorbidities, including hypertension, ischemic heart disease and diabetes mellitus, were additionally prevalent. At discharge, all patients were taking diuretics and the majority were taking beta-blockers, angiotensin-converting enzyme inhibitors and digoxin (Table I). Patients were hospitalized for a median length of 7 days (ranging between 1 and 38 days). Patients who succumbed within the 24-month follow-up exhibited a similar baseline profile compared with that observed in those who remained alive during this period; the only difference between the two groups of patients was the frequency of atrial fibrillation, which was more prevalent among those who died (Table I).

Plasma levels of BNP and miRs. Fig. 2 illustrates the plasma levels of BNP, miR-21, miR-126 and miR-423-5p in patients 
Table I. Baseline characteristics of patients with acute decompensated heart failure.

\begin{tabular}{|c|c|c|c|c|}
\hline \multirow[b]{2}{*}{ Characteristics } & \multirow[b]{2}{*}{ All patients $(n=48)$} & \multicolumn{2}{|c|}{ 24-month survival } & \multirow[b]{2}{*}{ P-value } \\
\hline & & Survived $(n=37)$ & Succumbed $(n=11)$ & \\
\hline Age, years & $62 \pm 13$ & $61 \pm 14$ & $64 \pm 10$ & 0.508 \\
\hline Male & $33(69 \%)$ & $25(68 \%)$ & $8(73 \%)$ & $>0.999$ \\
\hline Caucasian & $41(85 \%)$ & $32(86 \%)$ & $9(82 \%)$ & 0.653 \\
\hline \multicolumn{5}{|l|}{ Heart failure etiology } \\
\hline Ischemic & $18(38 \%)$ & $14(38 \%)$ & $4(36 \%)$ & 0.643 \\
\hline Hypertensive & $17(35 \%)$ & $11(30 \%)$ & $6(54 \%)$ & \\
\hline Alcoholic & $9(19 \%)$ & $8(22 \%)$ & $1(9 \%)$ & \\
\hline \multicolumn{5}{|l|}{ Clinical conditions at admission } \\
\hline NYHA functional class III/IV & $\begin{array}{l}15(31 \%) \\
33(69 \%)\end{array}$ & $\begin{array}{l}11(30 \%) \\
26(70 \%)\end{array}$ & $\begin{array}{l}4(36 \%) \\
7(64 \%)\end{array}$ & 0.720 \\
\hline Heart rate, bpm & $95 \pm 20$ & $95 \pm 20$ & $94 \pm 20$ & 0.852 \\
\hline Systolic blood pressure, $\mathrm{mmHg}$ & $130 \pm 28$ & $133 \pm 29$ & $122 \pm 24$ & 0.271 \\
\hline Diastolic blood pressure, $\mathrm{mmHg}$ & $85 \pm 17$ & $87 \pm 16$ & $79 \pm 18$ & 0.193 \\
\hline \multicolumn{5}{|l|}{ Comorbidities } \\
\hline Hypertension & $36(75 \%)$ & $27(73 \%)$ & $9(82 \%)$ & 0.705 \\
\hline Previous myocardial infarction & $17(35 \%)$ & $14(38 \%)$ & $3(27 \%)$ & 0.723 \\
\hline Diabetes mellitus & $24(50 \%)$ & $17(46 \%)$ & $7(64 \%)$ & 0.492 \\
\hline Chronic renal insufficiency & $11(23 \%)$ & $8(22 \%)$ & $3(27 \%)$ & 0.697 \\
\hline COPD & $18(38 \%)$ & $14(38 \%)$ & $4(36 \%)$ & $>0.999$ \\
\hline Smoking, past or current & $33(69 \%)$ & $25(68 \%)$ & $8(73 \%)$ & $>0.999$ \\
\hline Alcohol abuse, past or current & $23(48 \%)$ & $18(49 \%)$ & $5(46 \%)$ & $>0.999$ \\
\hline \multicolumn{5}{|l|}{ Echocardiographic data } \\
\hline LV ejection fraction, $\%$ & $26 \pm 8$ & $25 \pm 8$ & $26 \pm 10$ & 0.932 \\
\hline LV end-systolic diameter, $\mathrm{cm}$ & $5.7 \pm 0.9$ & $5.8 \pm 0.9$ & $5.6 \pm 1.1$ & 0.696 \\
\hline LV end-diastolic diameter, $\mathrm{cm}$ & $6.5 \pm 0.9$ & $6.5 \pm 0.8$ & $6.4 \pm 1.0$ & 0.564 \\
\hline \multicolumn{5}{|l|}{ Electrocardiogram data } \\
\hline Atrial fibrillation & $19(40 \%)$ & $11(30 \%)$ & $8(73 \%)$ & 0.016 \\
\hline Interventricular block & $19(40 \%)$ & $15(40 \%)$ & $4(36 \%)$ & $>0.999$ \\
\hline QRS duration, msec & $120 \pm 26$ & $122 \pm 24$ & $115 \pm 32$ & 0.451 \\
\hline \multicolumn{5}{|l|}{ Laboratory data } \\
\hline $\mathrm{BNP}$ at admission, $\mathrm{pg} / \mathrm{ml}$ & $659(472-1,255)$ & $667(480-1,333)$ & $566(374-1,075)$ & 0.593 \\
\hline Creatinine, $\mu \mathrm{mol} / \mathrm{l}$ & $115 \pm 35$ & $112 \pm 42$ & $114 \pm 33$ & 0.752 \\
\hline Urea, mmol/1 & $20.3 \pm 8.4$ & $19.3 \pm 8.4$ & $23.4 \pm 8.2$ & 0.136 \\
\hline Sodium, mmol/1 & $140 \pm 4$ & $139 \pm 4$ & $140 \pm 5$ & 0.394 \\
\hline Potassium, mmol/l & $4.2 \pm 0.4$ & $4.2 \pm 0.4$ & $4.2 \pm 0.5$ & 0.987 \\
\hline \multicolumn{5}{|l|}{ Medications at discharge } \\
\hline$\beta$-blockers & $38(79 \%)$ & $29(78 \%)$ & $9(82 \%)$ & $>0.999$ \\
\hline ACE inhibitors & $39(81 \%)$ & $31(84 \%)$ & $8(73 \%)$ & 0.409 \\
\hline Digoxin & $42(88 \%)$ & $32(89 \%)$ & $9(82 \%)$ & 0.609 \\
\hline Vasodilators & $24(50 \%)$ & $18(49 \%)$ & $6(54 \%)$ & $>0.999$ \\
\hline
\end{tabular}

Data are expressed as mean \pm standard deviation, median (25th-75th percentile), or n (\%). NYHA, New York Heart Association; COPD, chronic obstructive pulmonary disease; LV, left ventricular; BNP, B-type natriuretic peptide; ACE, angiotensin-converting enzyme.

with ADHF at the time of admission, discharge, and following clinical compensation. As hypothesized, BNP levels decreased between admission and discharge [Bonferroni-corrected P-value $\left.\left(P_{c}\right)<0.001\right]$ and compensation $\left(\mathrm{P}_{c}=0.003\right)$ (Fig. 2A).
The levels of miR-21 almost doubled between the time of admission and discharge (1.9-fold-change; $\left.\mathrm{P}_{c}=0.001\right)$, decreasing following clinical compensation $(0.65$-fold-change in relation to discharge; $\mathrm{P}_{c}=0.05$ ) (Fig. $2 \mathrm{~B}$ ). The levels of miR-126 

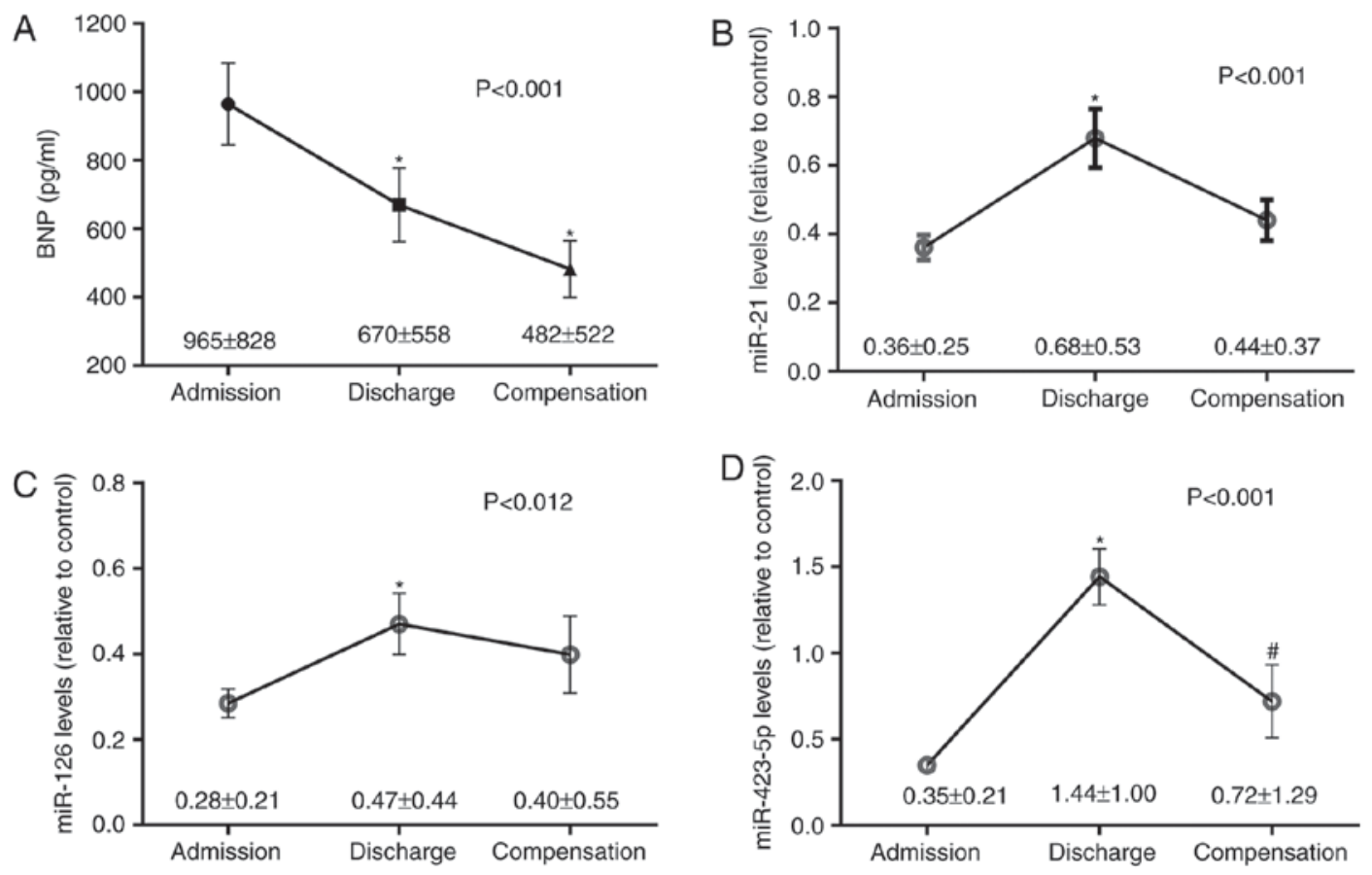

Figure 2. Measurement of plasma levels of BNP and miRs. Plasma levels of (A) BNP, (B) miR-21, (C) miR-126 and (D) miR-423-5p in patients with acute decompensated heart failure at the time of admission and discharge, and following clinical compensation. Levels are presented as the mean \pm standard error of the mean. ${ }^{*} \mathrm{P}<0.05$ vs. admission; ${ }^{~} \mathrm{P}<0.05$ vs. discharge. miR, microRNA; BNP, B-type natriuretic peptide.
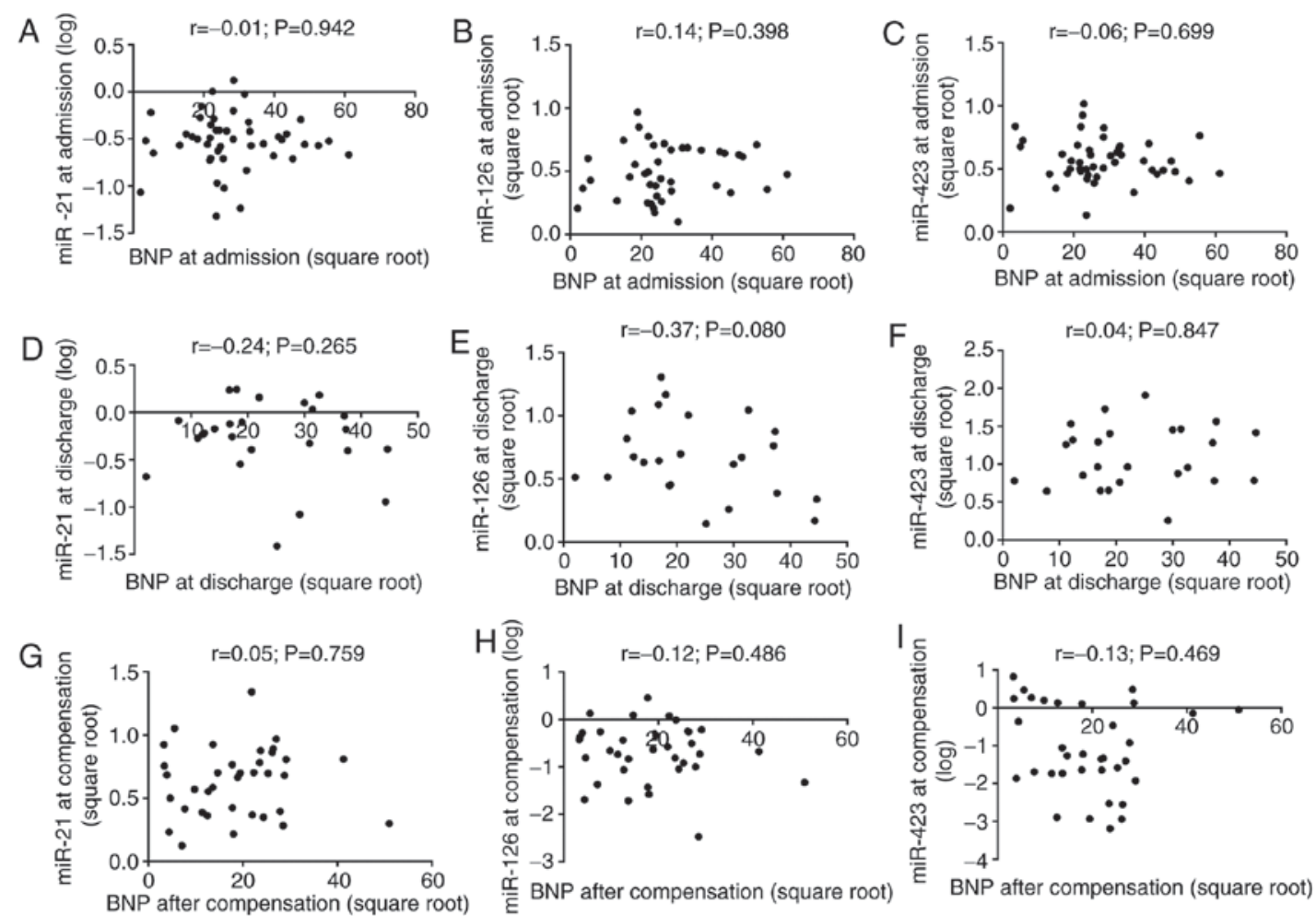

Figure 3. Scatterplots and Pearson correlation coefficients for relative expression levels of miRs with BNP levels in patients with acute decompensated heart failure. Relative levels of BNP with (A) miR-21, (B) miR-126, and (C) miR-423-5p at the time of admission and discharge (D-F) and following clinical compensation (G-I), respectively. miR, microRNA; BNP, B-type natriuretic peptide.

additionally increased between admission and discharge (1.7-fold-change; $\mathrm{P}_{c}=0.016$ ) (Fig. 2C). miR-423-5p expression exhibited the most prominent change, increasing between admission and discharge (4.1-fold-change; $\left.\mathrm{P}_{c}<0.001\right)$, and decreasing following clinical compensation (0.5-fold-change in relation to discharge; $\mathrm{P}_{c}=0.017$ ) (Fig. 2D).

As presented in Fig. 3, there was no correlation between the plasma levels of BNP and the three microRNAs at the time of 
admission and discharge, and following clinical compensation. The levels of miR-21 and miR-126 were directly correlated over time. At admission, miR-21 was positively correlated with miR-423-5p, whereas miR-126 was inversely correlated with miR-423-5p following clinical compensation (Table II). In addition, healthy controls had higher plasma microRNA levels in comparison with ADHF patients at admission and after clinical compensation (Fig. 4).

Association between miR levels, and hospital readmission and all-cause mortality. During the 4-year period following the initial admission, 38 patients $(79.2 \%)$ were rehospitalized at least once and 21 patients $(43.8 \%)$ succumbed. One-half of the patients had up to 36 months of follow-up treatment (ranging between 2 and 63 months). The number of hospital readmissions experienced by each patient varied between one $(n=7)$ and $42(n=1)$; almost $60 \%$ of the patients had up to three readmissions. In relation to all-cause mortality, the cumulative rates were, respectively, 23 and $44 \%$ at 24 and 48 months following initial admission.

A proportion of the patients had levels of BNP (65\%), miR-21 (43\%), miR-126 (52\%) and miR-423-5p (66\%) which reduced between admission and clinical compensation, while the remaining had exhibited increased levels between these two time points $(\Delta-\mathrm{BNP} / \Delta$-miR). The alteration in the plasma levels of BNP and miRs from initial admission to clinical compensation was not significantly different between patients who succumbed during the follow-up and those who remained alive (Table III). However, the levels of miR-21 and miR-126 at the time of clinical compensation were associated with all-cause mortality within 24 months following the initial admission. Notably, no patient with levels of miR-21 or miR-126 above the median succumbed (Fig. 5). Patients who succumbed within 24 months more frequently exhibited atrial fibrillation compared with those who remained alive (Table I), and patients with atrial fibrillation had 55\% lower miR-21 levels at the time of clinical compensation compared with those with sinus rhythm $(\mathrm{P}=0.008)$.

In addition, patients who had increased levels of miR-21 following clinical compensation remained rehospitalization-free for a longer time compared with those in whom the levels of miR-21 were below the median (mean period of 362 and 181 days, respectively; $\mathrm{P}=0.034$, for the first hospital readmission following the day of blood collection at the outpatient clinic). The same finding was observed for the levels of miR-126 (mean period of 423 and 177 days, respectively; $\mathrm{P}=0.028$ ). In addition, patients whose levels of miR-423-5p increased between admission and clinical compensation experienced fewer hospital readmissions in the 24-month period following the time of clinical compensation compared with those who had decreased levels (median of 0 and 2, respectively; $\mathrm{P}=0.040)$.

\section{Discussion}

The results of the present study demonstrated that the plasma concentrations of miR-21, -126 and -423-5p altered during the clinical improvement of patients with HF admitted for an episode of acute decompensation, and were associated with rehospitalization and all-cause mortality. A number of studies
Table II. Pearson correlation coefficients for the relative expression levels of miRs in patients with acute decompensated heart failure.

\begin{tabular}{lcc}
\hline Time point & miR-126 & miR-423-5p \\
\hline $\begin{array}{l}\text { At admission } \\
\text { miR-21 }\end{array}$ & $\mathrm{r}=0.54 ; \mathrm{P}<0.001$ & $\mathrm{r}=0.57 ; \mathrm{P}<0.001$ \\
miR-126 & - & $\mathrm{r}=-0.02 ; \mathrm{P}=0.913$ \\
$\begin{array}{l}\text { At discharge } \\
\text { miR-21 }\end{array}$ & $\mathrm{r}=0.76 ; \mathrm{P}<0.001$ & $\mathrm{r}=0.25 ; \mathrm{P}=0.126$ \\
miR-126 & - & $\mathrm{r}=0.02 ; \mathrm{P}=0.901$ \\
$\begin{array}{l}\text { Following clinical } \\
\text { compensation } \\
\text { miR-21 }\end{array}$ & & \\
miR-126 & $\mathrm{r}=0.70 ; \mathrm{P}<0.001$ & $\mathrm{r}=-0.26 ; \mathrm{P}=0.115$ \\
\hline
\end{tabular}

miR, microRNA.

have demonstrated that circulating miR levels in patients with stable or acute decompensated HF differ from those in control subjects, supporting the concept that they may be used as biomarkers for HF diagnosis $(6,8,14)$. The first evidence suggesting that circulating miRs may reflect HF severity was predominantly based on the observed correlations of the circulating miR levels with $\mathrm{BNP} / \mathrm{N}$-terminal prohormone $\mathrm{BNP}$ and/or other prognostic clinical parameters, including NYHA functional class and $\operatorname{LVEF}(9,15,16,23)$. Recently, miR levels were observed to be associated with rehospitalization and mortality in patients with acute HF (7,11-13). In the majority of these previous studies, circulating levels of miRs were measured at one or more time points, at most, within the first 7 days of the hospital admission, and the prognostic outcomes were evaluated after a short- or mid-term follow-up $(7,12,13)$.

The increase observed in the levels of miR-21 and miR-423-5p between hospital admission and discharge in the present study appears to contrast with the findings of previous studies into the clinical diagnosis of HF, in which the levels of miR-21 and miR-423-5p were increased in patients with HF compared with controls $(6,14)$. Based on this pattern, it may be hypothesized that the miR levels may be reduced during the clinical recovery of patients with ADHF. However, the results of the present study are in accordance with what is known about the pathophysiology of ADHF (17-19), with experimental evidence of the pathways regulated by miRs $(1-4,24)$, and with previous clinical studies into the prognosis of ADHF that investigated circulating levels of miR-21, miR-126 and miR-423-5p $(11,12)$. These findings suggested that patients considered clinically stable in a routine visit to the outpatient clinic, although exhibiting low levels of miR-21, miR-126 or miR-423-5p, may be more likely to experience new episodes of decompensation in the short-term.

miR-21 is expressed in cardiomyocytes, fibroblasts and endothelial cells, wherein it regulates apoptosis, fibrosis, and cell proliferation and migration, respectively (1). In the failing heart, overexpression of miR-21 in cardiac fibroblasts upregulates the RAC- $\alpha$ serine/threonine-protein kinase and 
Table III. Delta values of BNP and microRNAs according to all-cause mortality.

\begin{tabular}{lcccc}
\hline All-cause mortality & $\Delta$-BNP & $\Delta$-miR-21 & $\Delta$-miR-126 & $\Delta$-miR-423-5p \\
\hline Within 24 months & & & & \\
Yes (n=8) & $-328(-1605-19)$ & $-0.05(-0.17-0.15)$ & $-0.06(-0.19-0.01)$ & $0.01(-0.21-1.11)$ \\
No (n=32) & $-347(-935-208)$ & $0.11(-0.16-0.33)$ & $0.07(-0.10-0.38)$ & $-0.17(-0.30-0.57)$ \\
P-value & 0.710 & 0.507 & 0.146 & 0.284 \\
Within 48 months & & & $-0.05(-0.13-0.34)$ & $-0.15(-0.35-0.75)$ \\
Yes (n=18) & $-238(-1104-276)$ & $0.11(-0.15-0.47)$ & $0.07(-0.10-0.24)$ & $-0.16(-0.23-0.78)$ \\
No (n=22) & $-433(-1025-15)$ & $0.04(-0.16-0.29)$ & 0.515 & 0.643 \\
P-value & 0.664 & 0.465 & & \\
\hline
\end{tabular}

Data are presented as the median (25th-75th percentile). $\Delta$-BNP and $\Delta$-miRs represent the alteration in the plasma levels at the time of clinical compensation compared with initial admission. BNP, B-type natriuretic peptide; miR, microRNA.

A

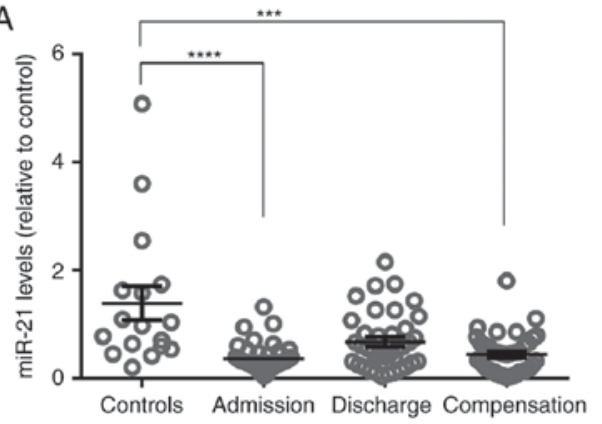

B

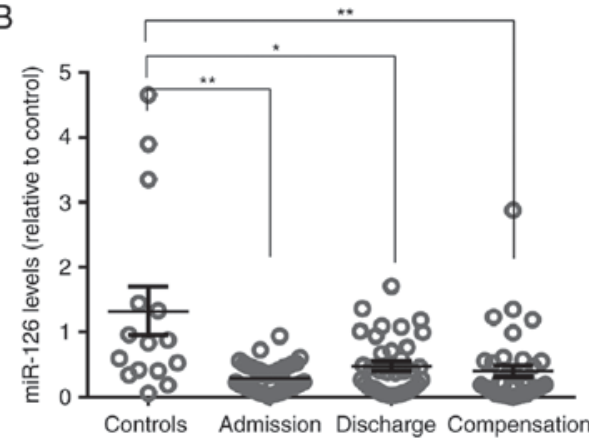

C

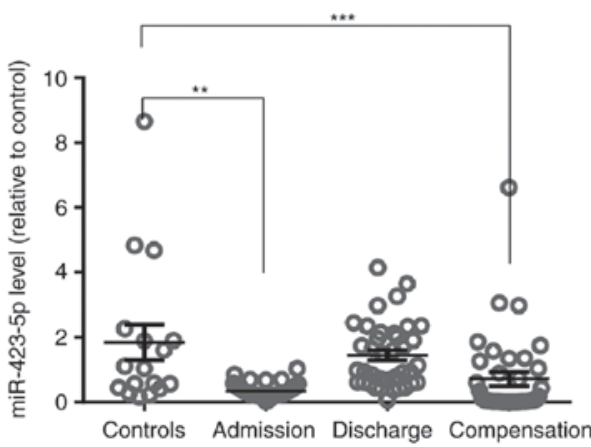

Figure 4. Analysis of plasma levels of miRs. Plasma levels of (A) miR-21, (B) miR-126 and (C) miR-423-5p in healthy controls compared with patients with acute decompensated heart failure at the time of admission and discharge, and following clinical compensation. Dispersion bars represent the standard error of the mean. ${ }^{*} \mathrm{P}<0.05 ;{ }^{* *} \mathrm{P}<0.01 ;{ }^{* * *} \mathrm{P}<0.001 ;{ }^{* * * *} \mathrm{P}<0.0001 . \mathrm{miR}$, microRNA.

mitogen-activated protein kinase signaling pathways, leading to fibroblast proliferation and fibrosis $(1,24,25)$, which in turn impairs ventricular function (1). However, miR-21 inhibits cellular apoptosis $(1,24,26)$. During myocardial ischemia, miR-21 is acutely downregulated within the ischemic zone, and replenishing its expression reduces the infarct size and delays the development of HF (26). These results were corroborated by experiments in cardiac myocytes, in which the overexpression of miR-21 was demonstrated to inhibit hypoxia-induced apoptosis, while the knockdown of miR-21 enhanced oxidative stress-induced apoptosis $(1,24)$. As the LV remodeling process involves myocyte loss by apoptosis (27), it may be hypothesized that in the initial clinical recovery (between admission and the time of hospital discharge), the increased levels of miR-21 observed in the present study contributed to protecting patients from acute myocyte loss during the episode of clinical decompensation.

In the present study, patients with high levels of miR-21 had an improved prognosis compared with those with reduced levels. In relation to rehospitalization, the results of the present study are consistent with those reported by Seronde et al (12), who assessed the plasma levels of miR-21, miR-126 and miR-423-5p in patients with acute HF. Levels of miR-21 were similar among patients with acute dyspnea (acute HF and non-acute HF) and patients with stable chronic HF. A subgroup of patients with acute HF had their miR levels measured at the time of admission and 5 days subsequently. Although the levels of miR-21 remained unaltered, they were decreased in patients who were readmitted in the year following the initial hospitalization compared with patients who were not. 
A

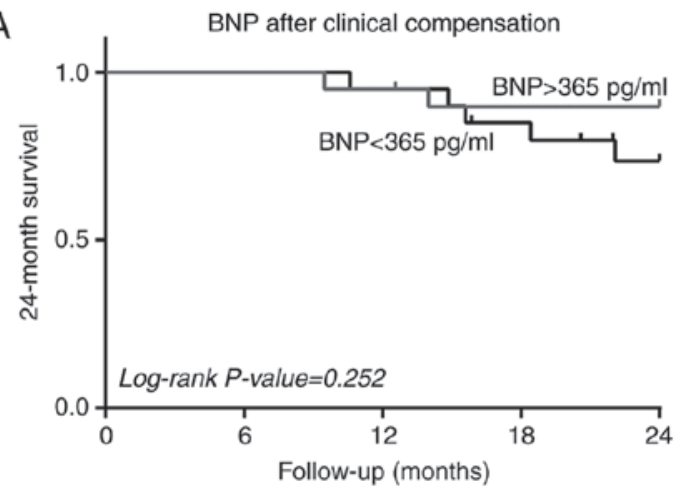

C

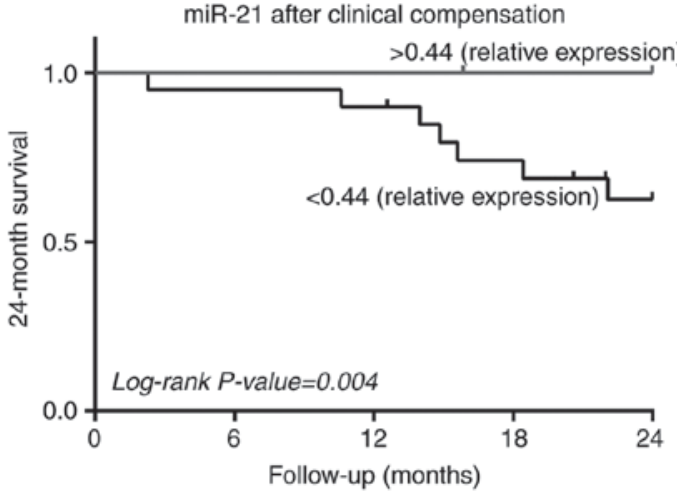

$\mathrm{E}$
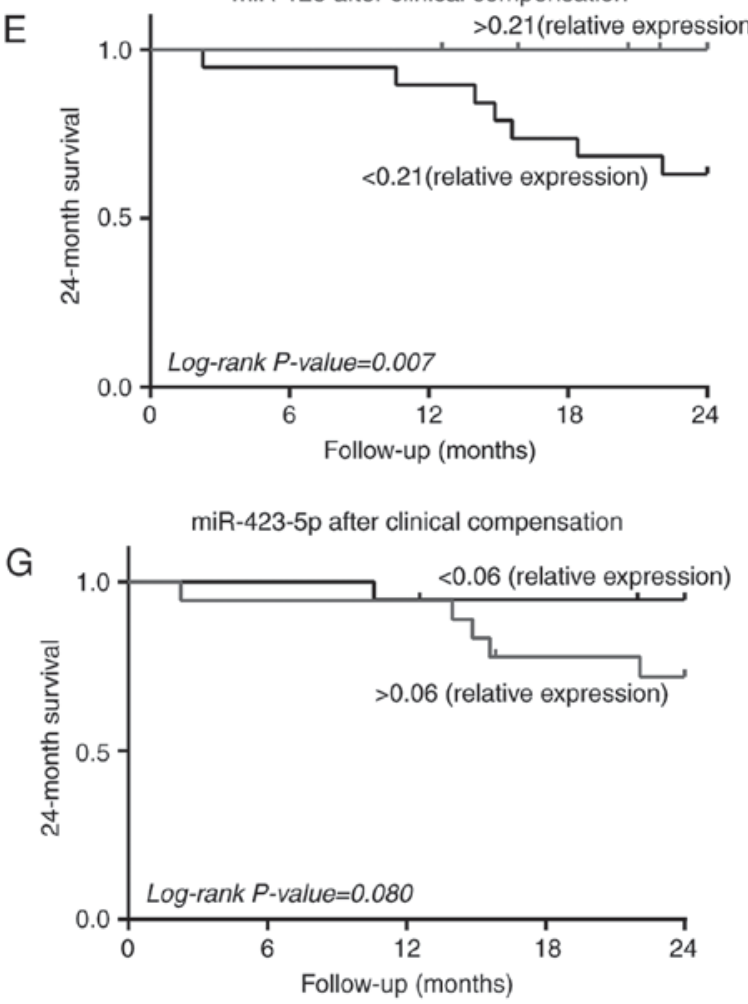

B

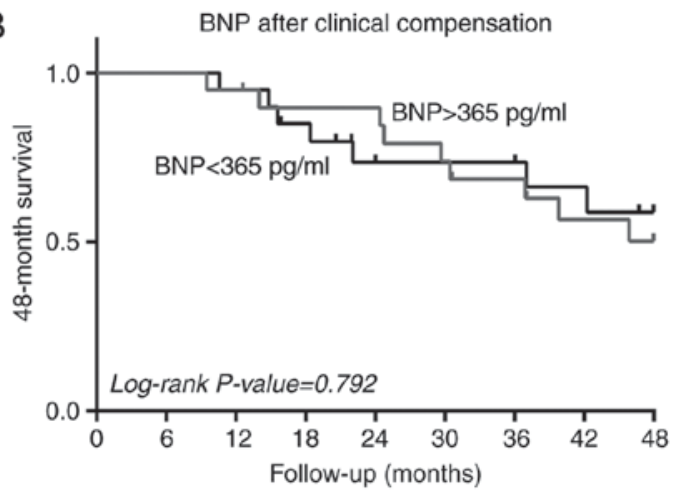

D

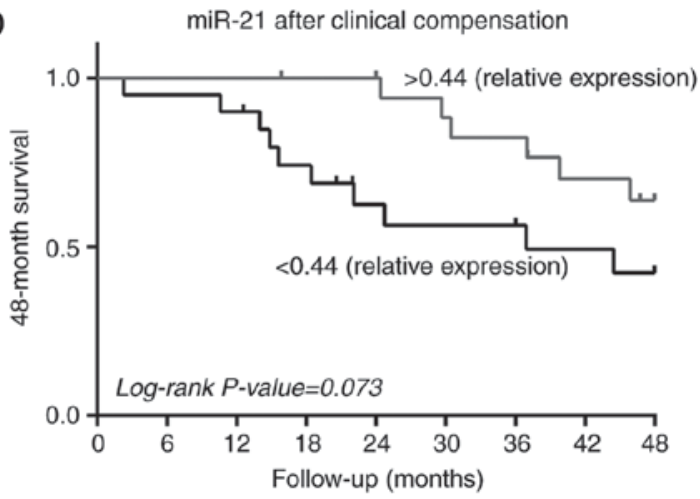

$\mathrm{F}$

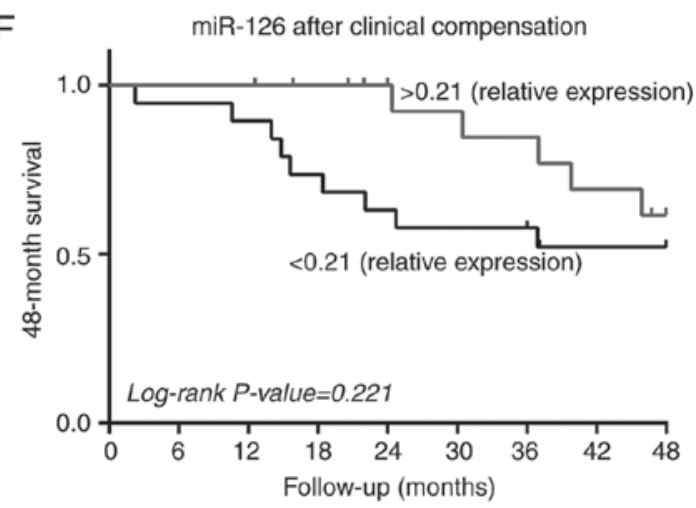

$\mathrm{H}$

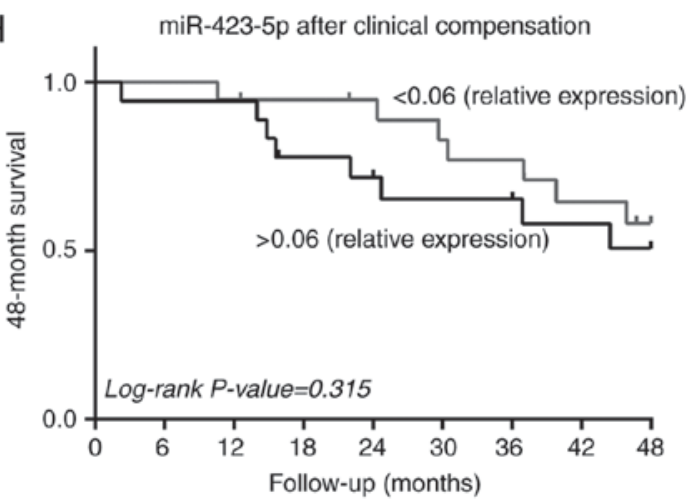

Figure 5. Kaplan-Meier survival curves for 24 and 48 -month mortality. Kaplan-Meier survival analysis was performed according to the levels of BNP at (A) 24 and (B) 48 months, miR-21 at (C) 24 and (D) 48 months, miR-126 at (E) 24 and (F) 48 months, and miR-423-5p at (G) 24 and (H) 48 months, at the time of clinical compensation. Survival curves illustrate the comparison between patients with plasma values above the median and those with plasma values below the median. miR, microRNA; BNP, B-type natriuretic peptide.

In relation to mortality, plasma levels of miR-21 were similar between patients who survived and patients who succumbed within the 1 year of follow-up. In the genome-wide expression study reported by Cakmak et al (7), plasma levels of miR-21 in patients with systolic HF were not associated with rehospitalization or cardiovascular mortality at 6 months of follow-up. In the present study, however, patients who succumbed during a 24-month follow-up period had 2 -fold lower plasma levels 
of miR-21 at the time of clinical compensation and more frequently exhibited atrial fibrillation compared with those who survived. The present results are in accordance with the findings reported by McManus et al (28) in a study population composed predominantly of middle-aged Caucasian men. The authors observed that subjects with atrial fibrillation had 2.1-fold lower plasma levels of miR-21 compared with controls, and that miR-21 expression increased by 3.4-fold following catheter ablation (28).

The endothelial cell-enriched miR-126 is highly expressed in the heart (26), where it maintains endothelial cell homeostasis and vascular integrity by enhancing angiogenesis. miR-126 is able to inhibit inflammation and atherosclerosis, leading to the recruitment of progenitor cells to repair the endothelial cells. In addition to its intracellular action, miR-126 exerts paracrine effects through the release of miR-126-containing microparticles, apoptotic bodies or exosomes $(1,2)$. In patients with $\mathrm{HF}$, impaired angiogenesis leads to alterations in the extracellular matrix that contribute to the LV remodeling process (27). As miR-126 is essential for ischemia-induced angiogenesis $(1,2)$, patients with HF may be expected to have lower circulating miR-126 levels. Indeed, it has been demonstrated that the circulating levels of miR-126 are decreased in patients with ischemic systolic HF compared with control subjects $(9,15)$, in addition to in patients with acute HF compared with those with stable HF (12). In 10 patients with HF, the plasma concentrations of miR-126 were assessed twice (when the patients were at NYHA functional class IV and when they improved to class III). As the clinical condition improved, plasma concentrations of miR-126 were upregulated (9). In rats with hypertension-induced HF, the plasma levels of miR-126 were increased in response to therapeutic treatment (29). In relation to prognosis, low levels of miR-126 were associated with readmission in the year following the initial hospitalization in acute HF (12), and with increased risk of cardiovascular death in patients with chronic HF of ischemic etiology followed-up for 24 months (8). These findings are similar to those obtained in the present study, as the levels of miR-126 increased between the time of initial admission and discharge, and patients with low levels of miR-126 had a worse prognosis compared with those with high levels.

A study by Tijsen et al (16) demonstrated that the plasma levels of miR-423-5p were able to be used to distinguish patients with HF from patients with dyspnea attributable to other causes and healthy controls. Similar results were observed in different populations, in which circulating miR-423-5p levels were increased in patients with HF compared with healthy subjects $(14,15,21,30-32)$. A previous study demonstrated a positive transcoronary gradient of plasma miR-423-5p in stable outpatients with HF, whereas a negative gradient was identified in subjects without structural cardiac disease, thereby suggesting that miR-423-5p may be of cardiac origin (33). Recently, the function of miR-423-5p began to be elucidated. Transfection experiments demonstrated that overexpression of miR-423-5p inhibited the proliferation and induced the apoptosis of human cardiomyoblasts (30) and murine cardiomyocytes via upregulation of p53 and caspase-3 (31).

However, in the context of the prognosis of acute HF in humans, decreased expression of miR-423-5p appears to be associated with the severity of HF and poor outcomes. In the report by Seronde et al (12), patients with acute dyspnea (with or without acute HF) had lower levels of miR-423-5p compared with those with stable HF. The levels upon admission of this miR were lower in patients who were readmitted to the hospital in the year following the initial hospitalization, compared with the patients who were not. In this same cohort, plasma levels of miR-423-5p were similar between patients who survived and those who succumbed within the 1 year of follow-up. In another cohort of patients with acute HF from the same study; however, low admission levels of miR-423-5p were associated with an increased risk of mortality (12). Similarly, circulating levels of 12 miRs, including miR-423-5p, were observed to be lower in acute $\mathrm{HF}$ (from three different cohorts) compared with chronic HF, acute exacerbation of chronic obstructive pulmonary disease and healthy controls (11). In one of the cohorts of acute HF, the levels of miRs were evaluated at four time points (at admission, and at $24 \mathrm{~h}, 48 \mathrm{~h}$ and 7 days following admission). In another cohort, their levels were measured at discharge and 6 months post-hospitalization. The lowest levels of miR-423-5p were observed in the period between admission and discharge. The levels increased and converged at 6 months towards the levels observed in patients with chronic $\mathrm{HF}$ and healthy controls. Additionally, further-decreasing levels of miR-423-5p within $48 \mathrm{~h}$ following hospital admission for acute HF were predictive of 180-day mortality (11). The results of the present study corroborated these previous studies $(11,12)$, as plasma levels of miR-423-5p increased from the time of admission until discharge, decreasing following clinical compensation. In the following 24 months, patients with low levels of miR-423 had a worse prognosis in terms of rehospitalization compared with those with high levels. These findings led to the hypothesis that miR-423-5p may reflect additional cardiomyocyte death occurring subsequent to multiple episodes of decompensation.

The findings of the present study and previous reports $(7,11,12)$ support the hypothesis that miR-21, -126 , and $-423-5 p$ may be useful as prognostic biomarkers of ADHF. Although the origin and the function of circulating miRs are not completely understood, an increasing number of studies have demonstrated that miRs are more than by-products of cellular injury, being actively secreted by cells and exerting paracrine effects $(34,35)$. However, it is important to understand that levels of plasma miRs fluctuate according to HF clinical status (stable chronic HF, ADHF admission, ADHF discharge and early compensated period), a concept that has not been considered in previous studies. In addition, pre-clinical studies in HF models revealed the potential of microRNA-based therapies to improve cardiac function, increase survival rates and reduce cardiac remodeling (34-36). Overall, this evidence suggested that circulating levels of miRs may help to identify patients who are at risk of poor outcomes and may even guide pharmacological therapy. However, findings from previous studies (7,11-13) and from the present cohort are insufficient to establish a cause and effect association. Consequently, it is too early to determine whether alterations in the expression of miRs during episodes of decompensation and clinical recovery are a consequence or a trigger of the functional and cellular alterations that occur in the failing myocardium.

The results of the present study may be interpreted in the light of certain logistical limitations. It was not possible to 
collect three blood samples from all patients included in the study, thus limiting the sample size and consequently the power of the observed associations. For the same reason, it was not possible to determine whether the associations detected were independent of clinical covariates, such as atrial fibrillation. Additionally, the selection of the three miRs investigated in the present study was based on intellectual choice rather than on genome-wide gene expression profiling. When the present study was designed, few reports had been published on circulating miRs in decompensated HF $(9,16)$. Among the miRs investigated in cardiovascular disorders, miR-21, miR-126 and miR-423-5p were recurrently observed to be dysregulated in atherosclerosis, acute coronary syndromes and HF (4-6). These miRs were selected as they are associated with cardiac myocyte injury, extracellular matrix turnover and inflammation (1-4,24), all of which are characteristic of HF decompensation (17-19). Despite these limitations, the present analysis is novel in the context of assessing the association between circulating miRs and the prognosis of ADHF in three different clinical scenarios in the same patients followed-up for a long period.

In conclusion, in the present prospective cohort study of patients admitted with ADHF, plasma levels of miR-21, miR-126 and miR-423-5p altered over time with clinical improvement, and low levels of these miRs were associated with poor outcomes (rehospitalization and all-cause mortality). The results of the present study confirm and expand the results from previous studies into ADHF, supporting the concept that plasma levels of miRs alter with HF progression and may have prognostic value.

\section{Acknowledgements}

The present study was supported by the Conselho Nacional de Desenvolvimento Científico e Tecnológico (CNPq, Brasília, Brazil; grant no. 478093/2011-0) and the Fundo de Incentivo à Pesquisa e Eventos do Hospital de Clínicas de Porto Alegre (Porto Alegre, Brazil; grant no. 11-0016). Dr Rohde received a research scholarship from CNPq (grant no. 308808/2013-4). The authors would like to thank Dr Fernanda Alves and Ms. Leticia Orlandin for assisting with sample and data collection.

\section{References}

1. Fang $\mathrm{YC}$ and $\mathrm{Yeh} \mathrm{CH}$ : Role of microRNAs in vascular remodeling. Curr Mol Med 15: 684-696, 2015.

2. Small EM and Olson EN: Pervasive roles of microRNAs in cardiovascular biology. Nature 469: 336-342, 2011.

3. Zhou J, Dong X, Zhou Q, Wang H, Qian Y, Tian W, $\mathrm{Ma} \mathrm{D}$ and Li X: MicroRNA expression profiling of heart tissue during fetal development. Int J Mol Med 33: 1250-1260, 2014.

4. Papageorgiou N, Tslamandris S, Giolis A and Tousoulis D: MicroRNAs in cardiovascular disease: Perspectives and reality. Cardiol Rev 24: 110-118, 2016.

5. Navickas R, Gal D, Laucevičius A, Taparauskaitė A, Zdanytė M and Holvoet P: Identifying circulating microRNAs as biomarkers of cardiovascular disease: A systematic review. Cardiovasc Res 111: 322-337, 2016.

6. Romaine SP, Tomaszewski M, Condorelli G and Samani NJ: MicroRNAs in cardiovascular disease: An introduction for clinicians. Heart 101: 921-928, 2015.

7. Cakmak HA, Coskunpinar E, Ikitimur B, Barman HA, Karadag B, Tiryakioglu NO, Kahraman K and Vural VA: The prognostic value of circulating microRNAs in heart failure: Preliminary results from a genome-wide expression study. J Cardiovasc Med (Hagestown) 16: 431-437, 2015.
8. Qiang L, Hong L, Ningfu W, Huaihong C and Jing W: Expression of miR-126 and miR-508-5p in endothelial progenitor cells is associated with the prognosis of chronic heart failure patients. Int J Cardiol 168: 2082-2088, 2013.

9. Fukushima Y, Nakanishi M, Nonogi $\mathrm{H}$, Goto $\mathrm{Y}$ and Iwai N: Assessment of plasma miRNAs in congestive heart failure. Circ J 75: 336-340, 2011.

10. Bruno N, ter Maaten JM, Ovchinnikova ES, Vegter EL, Valente MA, van der Meer P, de Boer RA, van der Harst P, Schmitter D, Metra M, et al: MicroRNAs relate to early worsening of renal function in patients with acute heart failure. Int J Cardiol 203: 564-569, 2016.

11. Ovchinnikova ES, Schmitter D, Vegter EL, Ter Maaten JM, Valente MA, Liu LC, van der Harst P, Pinto YM, de Boer RA, Meyer S, et al: Signature of circulating microRNAs in patients with acute heart failure. Eur J Heart Fail 18: 414-423, 2016.

12. Seronde MF, Vausort M, Gayat E, Goretti E, Ng LL, Squire IB, Vodovar N, Sadoune M, Samuel JL, Thum T, et al: Circulating microRNAs and outcome in patients with acute heart failure. PLoS One 10: e0142237, 2015.

13. Xiao J, Gao R, Bei Y, Zhou Q, Zhou Y, Zhang H, Jin M, Wei S, Wang K, Xu X, et al: Circulating miR-30d predicts survival in patients with acute heart failure. Cell Physiol Biochem 41: 865-874, 2017.

14. Fan KL, Zhang HF, Shen J, Zhang Q and Li XL: Circulating microRNAs levels in Chinese heart failure patients caused by dilated cardiomyopathy. Indian Heart J 65: 12-16, 2013.

15. Goren Y, Kushnir M, Zafrir B, Tabak S, Lewis BS and Amir O: Serum levels of microRNAs in patients with heart failure. Eur J Heart Fail 14: 147-154, 2012.

16. Tijsen AJ, Creemers EE, Moerland PD, de Windt LJ, van der Wal AC, Kok WE and Pinto YM: MiR423-5p as a circulating biomarker for heart failure. Circ Res 106: 1035-1039, 2010.

17. Biolo A, Fisch M, Balog J, Chao T, Schulze PC, Ooi H, Siwik D and Colucci WS: Episodes of acute heart failure syndrome are associated with increased levels of troponin and extracellular matrix markers. Circ Heart Fail 3: 44-50, 2010.

18. Sato Y, Yamada T, Taniguchi R, Nagai K, Makiyama T, Okada H, Kataoka K, Ito H, Matsumori A, Sasayama S and Takatsu Y: Persistently increased serum concentrations of cardiac troponin $\mathrm{T}$ in patients with idiopathic dilated cardiomyopathy are predictive of adverse outcomes. Circulation 103: 369-374, 2001.

19. Schulze PC, Biolo A, Gopal D, Shahzad K, Balog J, Fish M, Siwik D and Colucci WS: Dynamics in insulin resistance and plasma levels of adipokines in patients with acute decompensated and chronic stable heart failure. J Cardiac Fail 17: 1004-1011, 2011.

20. DeAguero JL, McKown EN, Zhang L, Keirsey J, Fischer EG, Samedi VG, Canan BD, Kilic A, Janssen PM and Delfín DA: Altered protein levels in the isolated extracellular matrix of failing human hearts with dilated cardiomyopathy. Cardiovasc Pathol 26: 12-20, 2017.

21. Thomé JG, Mendoza MR, Cheuiche AV, La Porta VL, Silvello D, Dos Santos KG, Andrades ME, Clausell N, Rohde LE and Biolo A: Circulating microRNAs in obese and lean heart failure patients: A case-control study with computational target prediction analysis. Gene 574: 1-10, 2015.

22. Livak KJ and Schmittgen TD: Analysis of relative gene expression data using real-time quantitative PCR and the 2(-Delta Delta C(T)) method. Methods 25: 402-408, 2001.

23. Endo K, Naito Y, Ji X, Nakanishi M, Noguchi T, Goto Y, Nonogi H, Ma X, Weng H, Hirokawa G, et al: MicroRNA 210 as a biomarker for congestive heart failure. Biol Pharm Bull 36: 48-54, 2013.

24. Wang J, Liew OW, Richards AM and Chen YT: Overview of microRNAs in cardiac hypertrophy, fibrosis, and apoptosis. Int J Mol Sci 17: E749, 2016.

25. Liu R-H, Ning B, Ma X-E, Gong WM and Jia TH: Regulatory roles of microRNA-21 in fibrosis through interaction with diverse pathways (Review). Mol Med Rep 13: 2359-2366, 2016.

26. Abdellatif M: Differential expression of microRNAs in different disease states. Circ Res 110: 638-650, 2012.

27. Topkara VK and Mann DL: Clinical applications of miRNAs in cardiac remodeling and heart failure. Per Med 7: 531-548, 2010.

28. McManus DD, Tanriverdi K, Lin H, Esa N, Kinno M, Mandapati D, Tam S, Okike ON, Ellinor PT, Keaney JF Jr, et al: Plasma microRNAs are associated with atrial fibrillation and change after catheter ablation (the miRhythm study). Heart Rhythm 12: 3-10, 2015. 
29. Dickinson BA, Semus HM, Montgomery RL, Stack C Latimer PA, Lewton SM, Lynch JM, Hullinger TG, Seto AG and van Rooij E: Plasma microRNAs serve as biomarkers of therapeutic efficacy and disease progression in hypertension-induced heart failure. Eur J Heart Fail 15: 650-659, 2013.

30. Li Q, Yu Q, Na R and Liu B: MiR-423-5p inhibits human cardiomyoblast proliferation and induces cell apoptosis by targeting Gab 1. Int J Clin Exp Pathol 9: 8953-8962, 2016.

31. Luo P, He T, Jiang R and Li G: MicroRNA-423-5p targets O-GlcNAc transferase to induce apoptosis in cardiomyocytes. Mol Med Rep 12: 1163-1168, 2015.

32. Olivieri F, Antonicelli R, Lorenzi M, D'Alessandra Y,Lazzarini R, Santini G, Spazzafumo L, Lisa R, La Sala L, Galeazzi R, et al: Diagnostic potential of circulating miR-499-5p in elderly patients with acute non ST-elevation myocardial infarction. Int J Cardiol 167: 531-536, 2013
33. Goldraich LA, Martinelli NC, Matte U, Cohen C, Andrades M, Pimentel M, Biolo A, Clausell N and Rohde LE: Transcoronary gradient of plasma microRNA 423-5p in heart failure: Evidence of altered myocardial expression. Biomarkers 19: 135-141, 2014.

34. Vegter EL, van der Meer P, de Windt LJ, Pinto YM and Voors AA: MicroRNAs in heart failure: From biomarker to target for therapy. Eur J Heart Fail 18: 457-468, 2016.

35. Viereck J, Bang C, Foinquinos A and Thum T: Regulatory RNAs and paracrine networks in the heart. Cardiovasc Res 102: 290-301, 2014.

36. Caroli A, Cardillo MT, Galea R and Biasucci LML: Potential therapeutic role of microRNAs in ischemic heart disease. J Cardiol 61: 315-320, 2013. 